\title{
Is disturbed transfer of learning in callosal agenesis due to a disconnection syndrome?
}

\author{
T. Imamura ${ }^{1}$, A. Yamadori' ${ }^{2}$ Y. Shiga ${ }^{1}$, M. Sahara ${ }^{3}$ and H. Abiko ${ }^{4}$ \\ ${ }^{1}$ Department of Neurology, Institute of Brain Diseases, Tohoku University School of Medicine, \\ Sendai, ${ }^{2}$ Hyogo Institute for Aging Brain and Cognitive Disorders, Himeji, ${ }^{3}$ Department of \\ Neurology, Kohnan Hospital, Sendai, and ${ }^{4}$ Department of Neurosurgery, National Sendai \\ Hospital, Sendai, Japan \\ Correspondence to: T. Imamura, Division of Behavioral Neurology, Department of Clinical \\ Neurosciences, Hyogo Institute for Aging Brain and Cognitive Disorders, 520, Saisho-Ko, \\ Himeji 670, Japan
}

\begin{abstract}
Disturbed intermanual transfer of tactile learning in callosal agenesis has been interpreted as a sign of disconnection syndrome. We observed this sign in one of four acallosal patients with a conventional form-board task, and tried to elucidate the nature of the deficit. The form-board performance of the patient with disturbed transfer of learning totally depended on motor skill, while the other acallosals and normal controls executed the task based on spatial and somesthetic information. All acallosals and normals, however, failed to show transfer of learning with another tactile task which needed motor skill but not spatialsomesthetic information. These findings suggest that the task-performing strategies in form-board learning change the state of interhemispheric transfer. Unimanual learning effect is transferred if spatial-somesthetic information is acquired in the process of learning, but is not transferred if motor skill is the exclusive content of learning. We conclude that disturbed "transfer" of learning in some acallosals is not a true disconnection sign. It should be attributed to a lack of appropriate strategy, as a result of ineffective problem solving in tactile tasks.
\end{abstract}

Keywords: Corpus callosum - Callosal disconnection - Malformation - Problem solving

\section{INTRODUCTION}

Computed tomography (CT) and magnetic resonance imaging (MRI) have increased the chances of diagnosing callosal agenesis. On neurobehavioral examination, acallosal patients exhibit less callosal syndromes than acquired disconnections (Bogen, 1985; Jeeves, 1990). Disturbed transfer of learning, however, occurs frequently. Acallosals have difficulties after unimanual learning on a tactile task, such as formboard (Russell and Reitan, 1955; Solursh et al., 1965; Ferriss and Dorsen, 1975; Reynolds and Jeeves, 1977; Gott and Saul, 1978; Jeeves, 1979; Soma et al., 1982) or stylus maze (Lehmann and Lampe, 1970; Ferriss and Dorsen, 1975; Gott and Saul, 1978; Jeeves, 1979), in transferring information to the contralateral hand. This may be due to disturbed interhemispheric transfer of "experience" or "training" (Russell and Reitan, 1955; Reynolds and Jeeves, 1977), or impaired transfer of complex somesthetic or spatial information (Lehmann and Lampe, 1970; Gott and Saul, 1978; Soma et al., 1982). We have studied four acallosal patients using two kinds of tactile tasks.

\section{METHODS}

\section{Patients}

Four right-handed patients with callosal agenesis

TABLE I. Patients' characteristics

\begin{tabular}{|c|c|c|c|c|c|c|c|}
\hline \multirow[t]{2}{*}{ Patient No. } & \multirow[t]{2}{*}{ Age (years) } & \multirow[t]{2}{*}{ Sex } & \multirow[t]{2}{*}{ Medical history } & \multirow[t]{2}{*}{ Neurological findings } & \multicolumn{3}{|c|}{ Scores on WAIS } \\
\hline & & & & & VIQ & $\mathrm{PIQ}$ & TIQ \\
\hline 1 & 47 & M & None & None & 78 & 100 & 86 \\
\hline 2 & 73 & $M$ & None & None & 92 & 69 & 83 \\
\hline 3 & 44 & $\mathrm{~F}$ & Paranoid disorder & None & 60 & 81 & 67 \\
\hline 4 & 68 & M & Left elbow joint trauma & Clumsiness of left hand & 70 & 86 & 74 \\
\hline
\end{tabular}




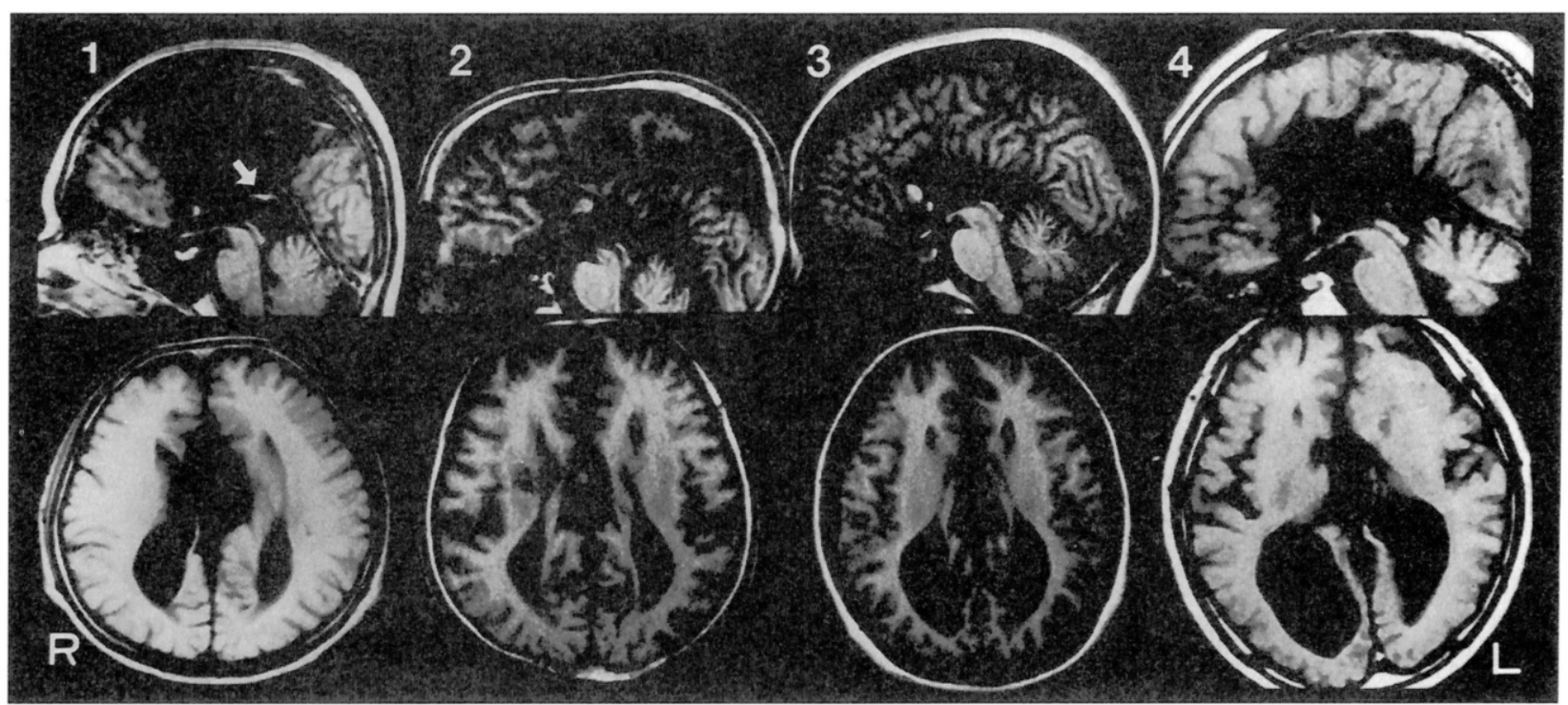

FIG. 1. Sagittal (top) and axial (bottom) MRI revealed callosal agenesis and associated anomalies of patients. Arabic numerals indicate patient numbers. In sagittal image of Patient 1 , the wall of the subarachnoid cyst resembles corpus callosum (arrow).

were studied. They all lived independent social lives, and their age, sex, medical history, present neurological findings and scores on the Wechsler Adult Intelligence Scale (WAIS) are presented in Table I. The left hand of Patient 4 was somewhat clumsy because of limited flexions of the 3rd, 4th and 5th fingers, which was the sequelae of elbow joint trauma in his youth. MRI demonstrated callosal agenesis and associated findings, i.e. parasagittal subarachnoid cysts in Patients 1 and 4 , and a minimal residual corpus callosum in Patients 2 and 3 (Fig. 1). The presence of an anterior commissure was confirmed in all patients.

On neuropsychological assessment, no patients had aphasia or visual agnosia. There was no agraphia, tactile anomia, spatial neglect, constructional disturbance or ideomotor apraxia in either hand. Crossed tactile identification was normal and crossed visuomotor ataxia was negative. Crossed point localization, assessed by the reported method (Bogen, 1985), was normal in Patient 4, but impaired in the other patients. All of them showed normal bimanual coordination, and no diagnostic dyspraxia or alien hand syndrome (Bogen, 1985; Feinberg et al., 1992). Tachistoscopical examinations for visual half field recognition and dichotic listening were performed in Patients 1 and 4, and revealed no signs of callosal disconnection.

\section{Tasks for tactile learning}

Form-board task. A conventional form-board task was given to the patients and to four right-handed normal volunteers age matched with Patients 1 and 3. This task consisted of four wooden forms and a board with four holes. Each subject was blindfolded and then instructed to put the forms to the corresponding holes as quickly as possible. The forms and board were placed in front of a subject (Fig. 2, left), then an examiner guided the subject's hand over the forms and the board. The examiner ensured that the subject could assess the distance but could not perceive the shape of each form or the spatial arrangement of holes during this instruction. The task was started with the non-preferred hand and repeated until the performance time reached a negative plateau, then the same procedure was repeated with the preferred hand, except in Patient 4 who began with the right hand against instruction. The performance time of each trial was measured manually, and normalized as a ratio to the first trial of the initial hand. The examiner observed and noted the strategies used by each subject.

Finger manipulation task. This task examined the unimanual learning of motor skill and its transfer in Patients 1-3 and eight right-handed normal subjects. Normal Controls 1-4 and 5-8 were age matched with Patients 1 and 3 and Patient 2, respectively. Patient 4 was excluded from this task because of his clumsy left fingers. Each subject was required, with a single hand, to open the cap of a transparent plastic cylinder which had a stick inside (Fig. 2a-c). An examiner instructed each subject how to: (a) hold the cylinder in one hand; (b) open the cap with the thumb and index finger and drop the stick to the desk; and (c) put the cap on again, with visual and verbal presentation. Thus, the subjects acquired the visuospatial

44 Behavioural Neurology . Vol 7 . 1994 


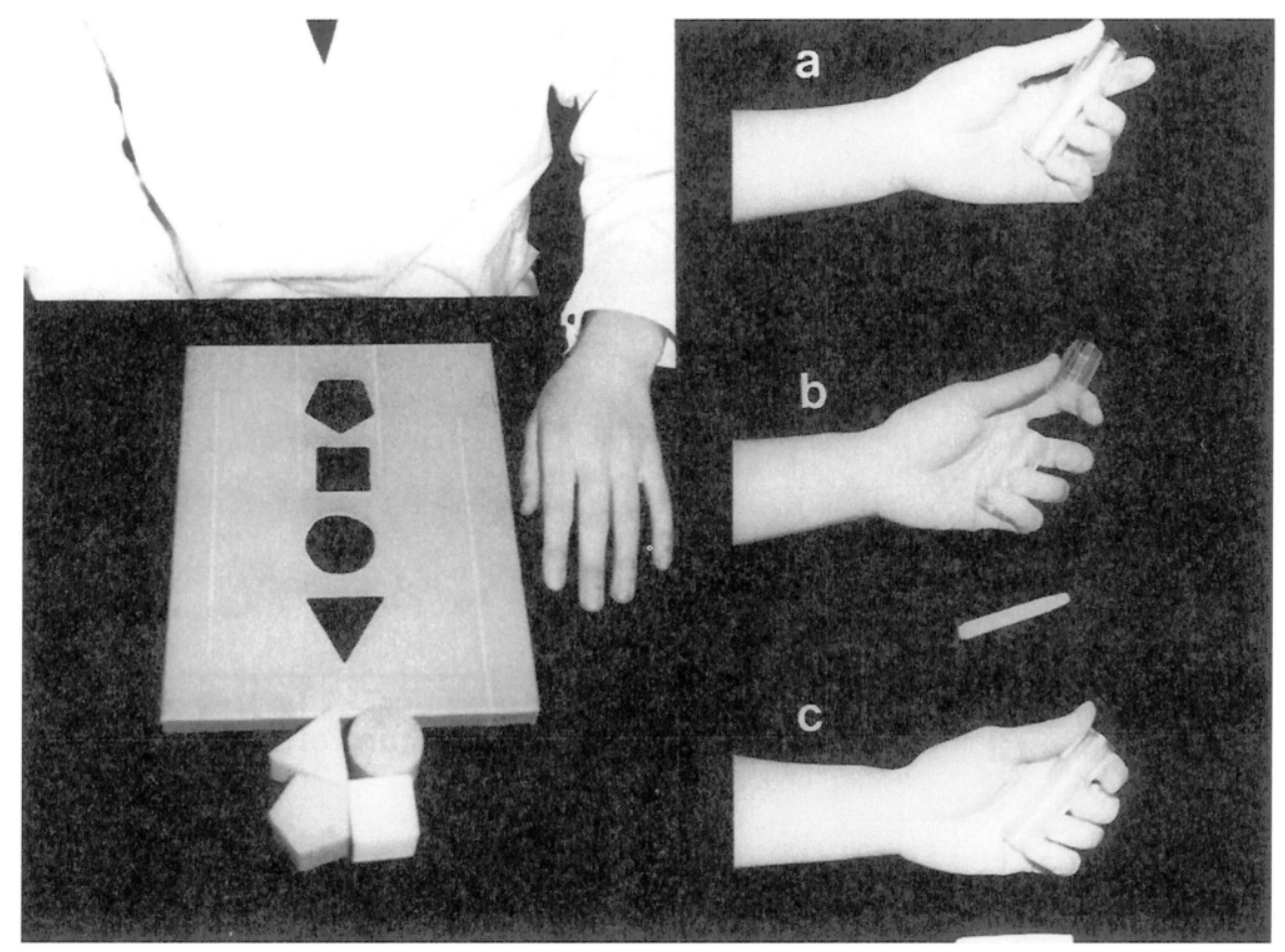

FIG. 2. Form-board task (left) and finger manipulation task (right).

TABLE II Subjects' strategy and transfer of learning

\begin{tabular}{cccccc}
\hline Patients & \multicolumn{2}{c}{ Form-board task } & & \multicolumn{2}{c}{ Finger manipulation task } \\
\cline { 2 - 3 } \cline { 5 - 6 } & Strategy & Transfer & & Strategy & Transfer \\
\hline 1 & Form selection & + & & Same as instruction \\
2 and 3 & Direct & + & & Same as instruction & (Not done) \\
4 & Trial and error & - & & Same as instruction \\
Normal controls & Direct & + & & Sam
\end{tabular}

information for this task before the actual performance, and they only needed to learn unimanual motor skill for tactile manipulation. With a blindfold in place, the task was started with the non-preferred hand and repeated to the negative plateau of performance time, then the preferred hand repeated the same procedure. Performance time was measured and normalized just as the form-board task, and the subjects' strategies were recorded.

\section{RESULTS}

\section{Form-board task (Fig. 3 and Table II)}

The performance time ratio of the initial hand steadily decreased, and demonstrated a definite learning effect in all subjects. No such decrease appeared in the contralateral hand of Patients 1-3 and the normal controls, i.e. the performance time ratio started from the lower rate and remained at the same level through- out the trials. Thus, we concluded that the learning effect of the initial hand was transferred to the other hand in Patients 1-3. In Patient 4, performance time of the left hand was prolonged because of his motor impairment. It is, nevertheless, safe to say that the decline in performance time revealed comparable learning effects in both hands. With regard to the performance time, we could not detect intermanual transfer of learning in Patient 4.

The subjects' strategy was not uniform. In the first trial of the initial hand, all subjects picked up one form and tried to put it into one hole in a random manner. This "trial and error" strategy soon altered in Patients 2 and 3, as well as in all the normal subjects. After several trials, they could put any form directly into the corresponding hole with no hesitation. This "direct" strategy was transferred to the other side, i.e. the contralateral hand employed this strategy from the start. Patient 1 acquired another 

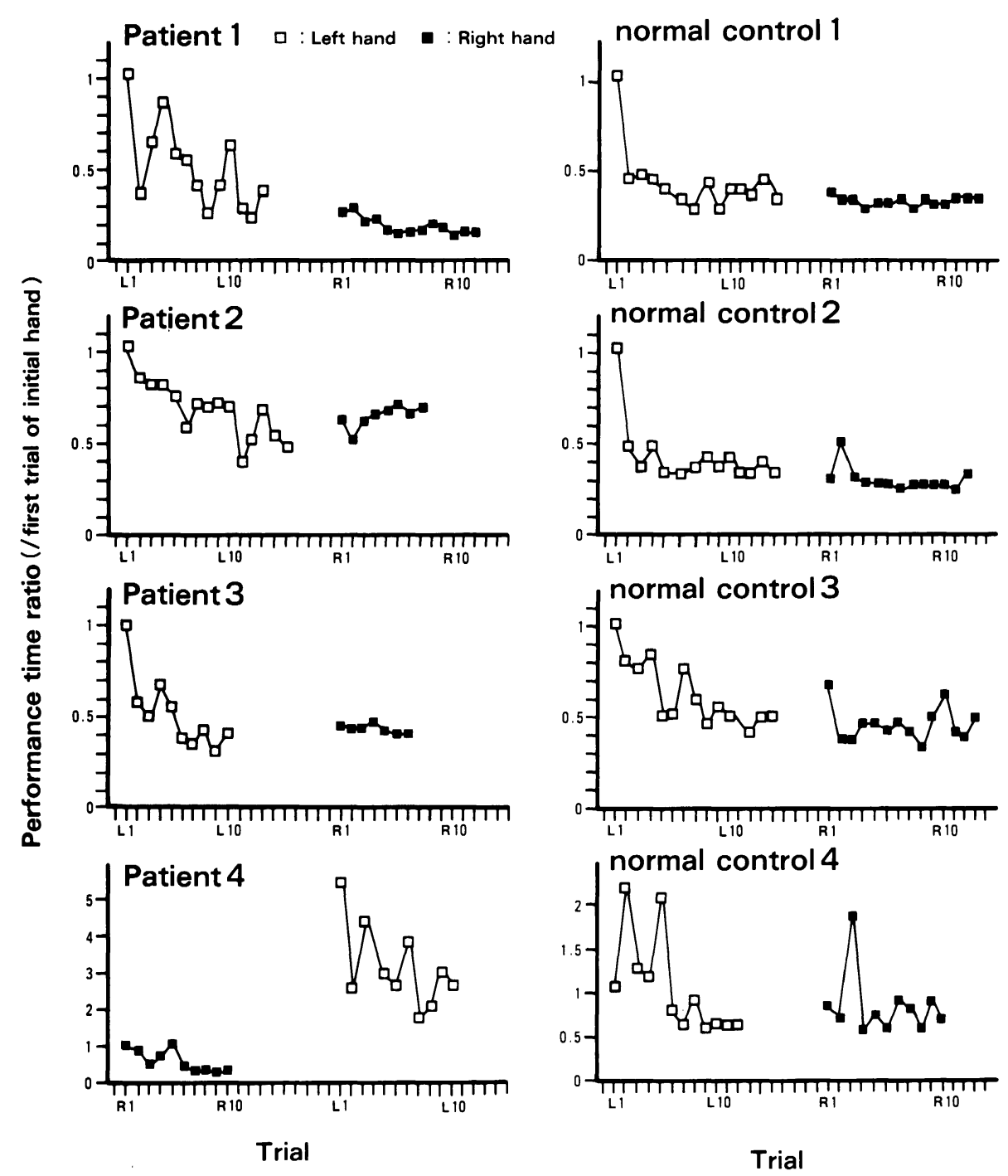

FIG. 3. Subjects' performance time on form-board task.

effective strategy, in which he first groped for the triangle and put it into the top hole, and then groped for and put the circle, square and pentagon into the 2nd, 3rd and 4th holes (see Fig. 2, left). This "form selection" strategy also transferred to the contralateral hand. Patient 4 could not find any effective strategy and performed all trials of both hands in a "trial and error" manner.

\section{Finger manipulation task (Fig. 4 and Table II)} In some trials, the subjects' attention was distracted by the stick falling onto the floor. We discarded the performance time of these trials, and indicated them as a blank entry in Fig. 4. In Patients 1-3 and normal Controls $3-8$, the performance time clearly declined in both hands, which revealed comparable learning effects. In normal Controls 1 and 2 there was no demonstrable learning effect in either hand. All subjects performed the task following the examiner's instruction, and no one changed the strategy throughout the trials. Intermanual transfer of motor skill did not occur either in patients or normal subjects.

\section{DISCUSSION}

The patients' clinical and radiological findings were consistent with callosal agenesis (Bogen, 1985; Jeeves, 1990). Their neurological function was intact except for a coincidental impairment in Patient 4. Neuroimaging revealed a combination of absent corpus callosum, persistent anterior commissure, and some associated anomalies. On neuropsychological examination, a discrepancy of verbal and performance IQs was apparent on WAIS, and some patients showed im- 

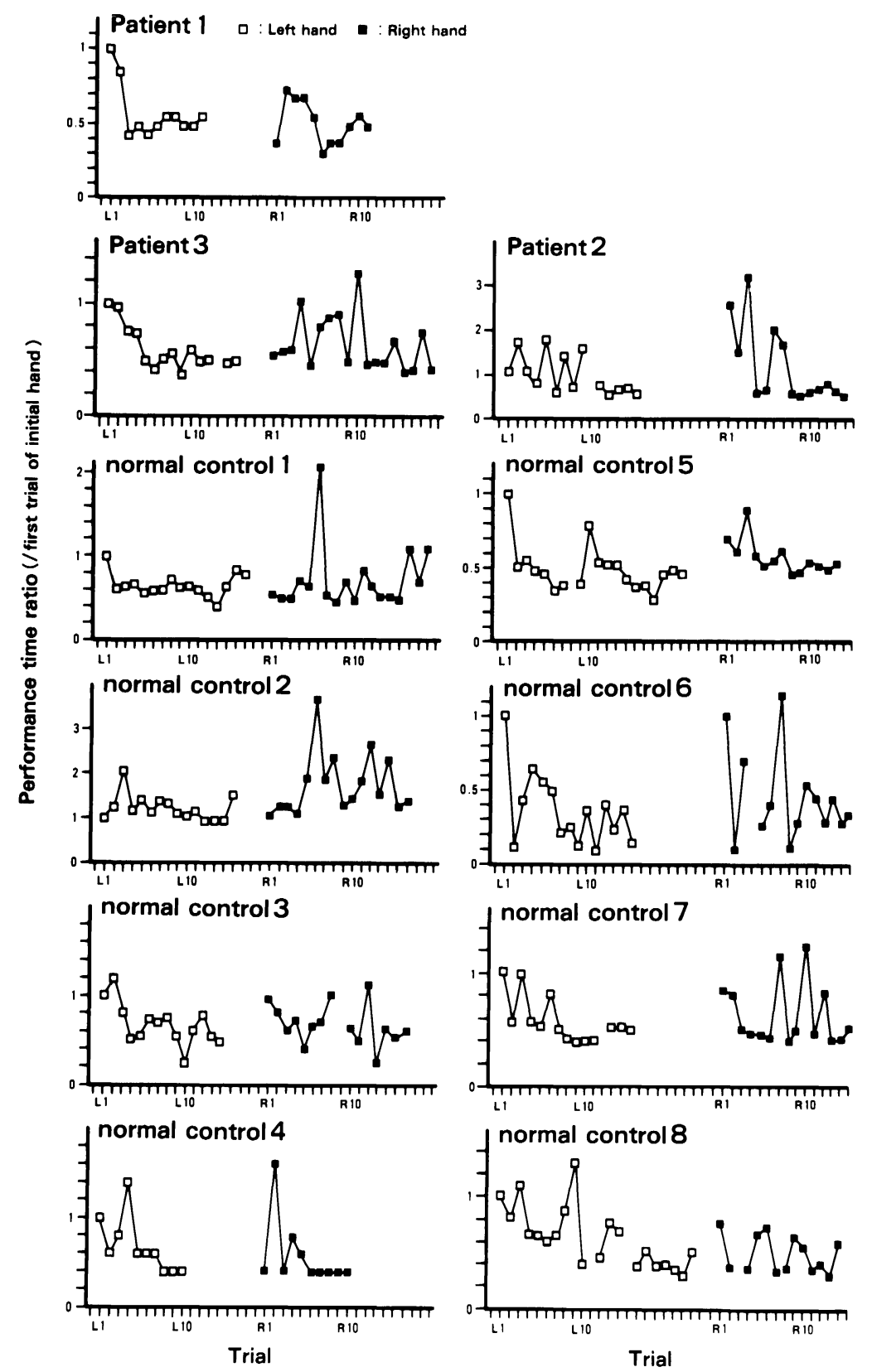

FIG. 4. Subjects' performance time on finger manipulation task. Blanks indicate trials in which the subject's attention was disturbed (see text).

paired crossed point localization. Disturbed transfer of learning was detected in Patient 4 on the formboard task.

The observations relating to the strategies used to perform the form-board task (Table II) revealed how the subjects acquired and transferred information. The "direct" and "form selection" strategies require the knowledge of the spatial arrangement of holes and the shapes of forms. Spatial and somesthetic information was successfully retained, stored, and transferred to the contralateral hand in Patients 1-3 and normal subjects. Patient 4 did not employ these spatial-somesthetic strategies, and his performance was a result of trial and error. These findings suggest that both normal and acallosal subjects can transmit acquired spatial-somesthetic information, a finding which contradicts the previous view which attributed disturbed transfer of form-board learning in acallosals to the disconnection of spatial-somesthetic information between hemispheres (Gott and Saul, 1978; 
Soma et al., 1982). Learning in Patient 4, who did show disturbed transfer of learning, depended solely on motor skill for tactile manipulation, raising the possibility that disconnection of motor skill might explain disturbed learning transfer. However, the unimanual learning effect was not transferred in any subjects including normals. This indicates that neither normal nor acallosal neural systems are able to transmit motor skill for tactile manipulations.

Our observations indicate that the disturbed transfer of form-board learning in Patient 4 is attributed to his strategy. His "trial and error" manner depends on motor skill, which cannot be transferred and must be acquired separately in each hand. On the other hand, learning was transferred in the other subjects because they employed spatial-somesthetic strategies. Thus, we conclude that the disturbed transfer of learning in callosal agenesis is a result of inadequate strategy that does not utilize spatial-somesthetic information. In other words, it is a manifestation of ineffective problem solving. The descriptions of Ferriss and Dorsen (1975) are consistent with this assumption. Their acallosal patients, who manifested disturbed transfer of learning, were unable to assess the shape or size of forms and persisted in trial and error placement on a form-board task. In their performances of stylus maze, there was no reduction of error score, and any saving in time was due to a lesser dwelling time and faster movements. Jeeves (1990) also assumed some intrahemispheric deficits, rather than callosal disconnection, for the mechanism of disturbed transfer of learning. The origin of this disability is uncertain, but it is possible that associated brain anomalies may be responsible for some of the observed cognitive impairment (Jeeves, 1990).

\section{REFERENCES}

Bogen JE (1985) The callosal syndromes. In: Clinical Neuropsychology, 2nd edn (Eds KM Heilman and E Valenstein), pp. 295-338. Oxford University Press, New York.

Feinberg TE, Schindler RJ, Flanagan NG and Haber LD (1992) Two alien hand syndromes. Neurology, 42, 19-24.

Ferriss GS and Dorsen MM (1975) Agenesis of the corpus callosum: 1. Neuropsychological studies. Cortex, 11, 95122.

Gott PS and Saul RE (1978) Agenesis of the corpus callosum: Limits of functional compensation. Neurology, 28, 1272-1279.

Jeeves MA (1979) Some limits to interhemispheric integration in cases of callosal agenesis and partial commissurotomy. In Structure and Function of Cerebral Commissures, pp. 449-474. MacMillan Press, London.

Jeeves MA (1990) Agenesis of corpus callosum. In: F Boller and J Grafman (Eds), Handbook of Neuropsychology, Vol 4 (Eds IS Russell, MW van Hof and G Berlucchi), pp. 99-114. Elsevier Science Publishers, New York.

Lehmann HJ and Lampe H (1970) Observations on the interhemispheric transmission of information in 9 patients with corpus callosum defect. European Neurology, 4, 129-147.

Reynolds DM and Jeeves MA (1977) Further studies of tactile perception and motor coordination in agenesis of the corpus callosum. Cortex, 13, 257-272.

Russell JR and Reitan RM (1955) Psychological abnormalities in agenesis of the corpus callosum. Journal of Nervous and Mental Diseases, 121, 205-214.

Solursh LP, Margulies AI, Ashem B and Stasiak EA (1965) The relationships of agenesis of the corpus callosum to perception and learning. Journal of Nervous and Mental Diseases, 141, 180-189.

Soma Y, Sugishita M, Iwata M and Hirota K (1982) Agenesis of the corpus callosum: A neuropsychological study. Clinical Neurology (Tokyo), 22, 526-533 (in Japanese). 


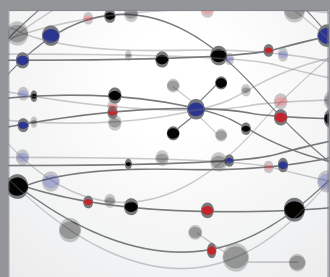

The Scientific World Journal
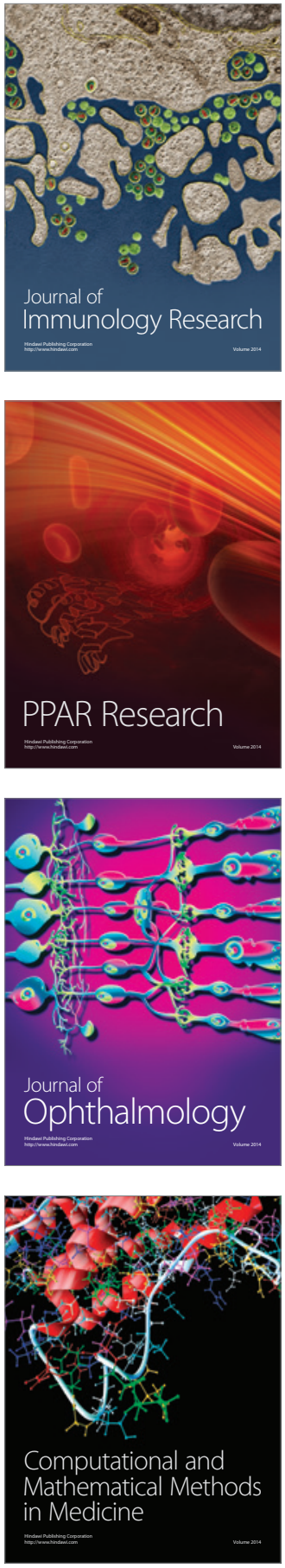

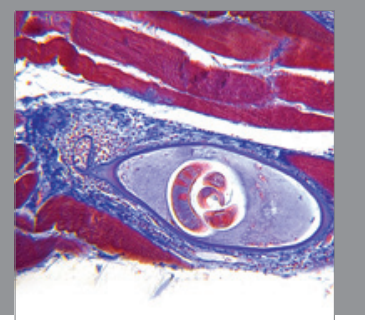

Gastroenterology

Research and Practice
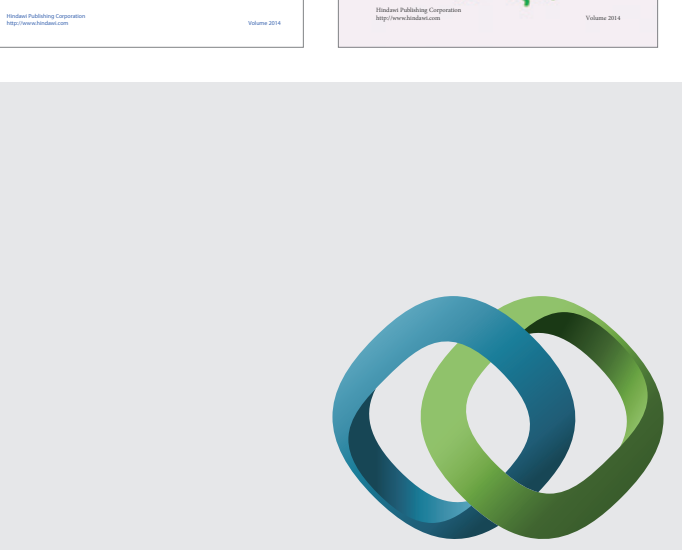

\section{Hindawi}

Submit your manuscripts at

http://www.hindawi.com
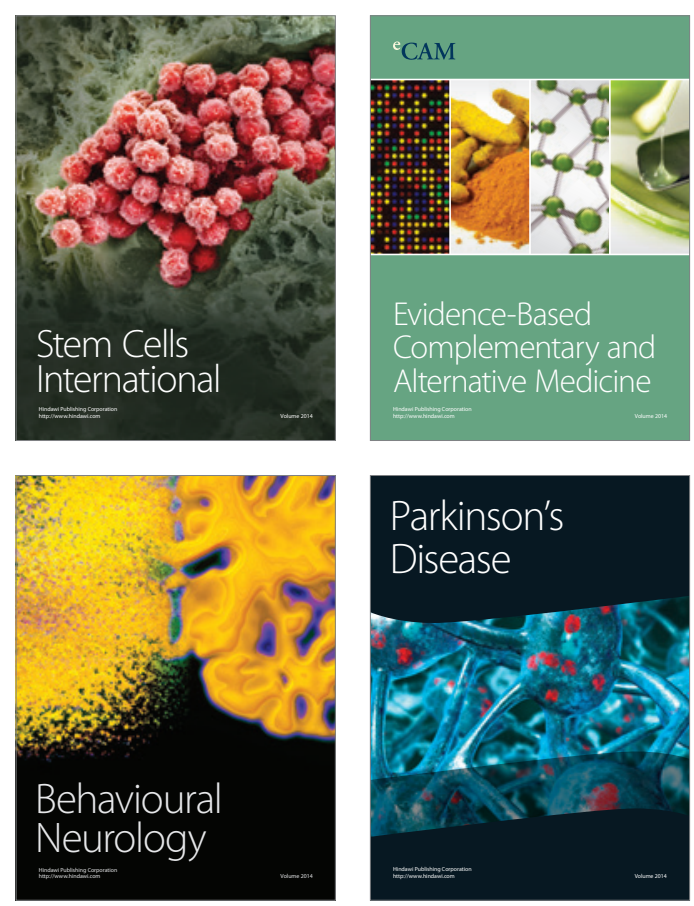

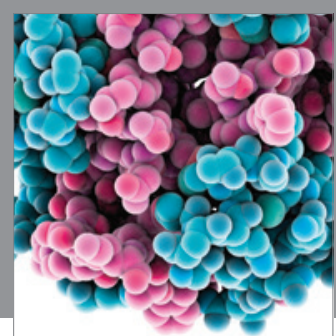

Journal of
Diabetes Research

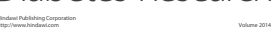

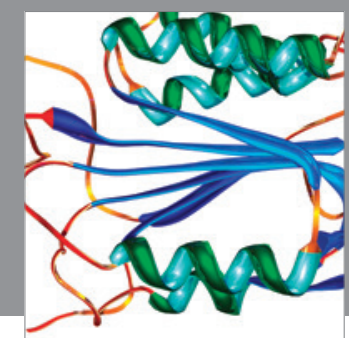

Disease Markers
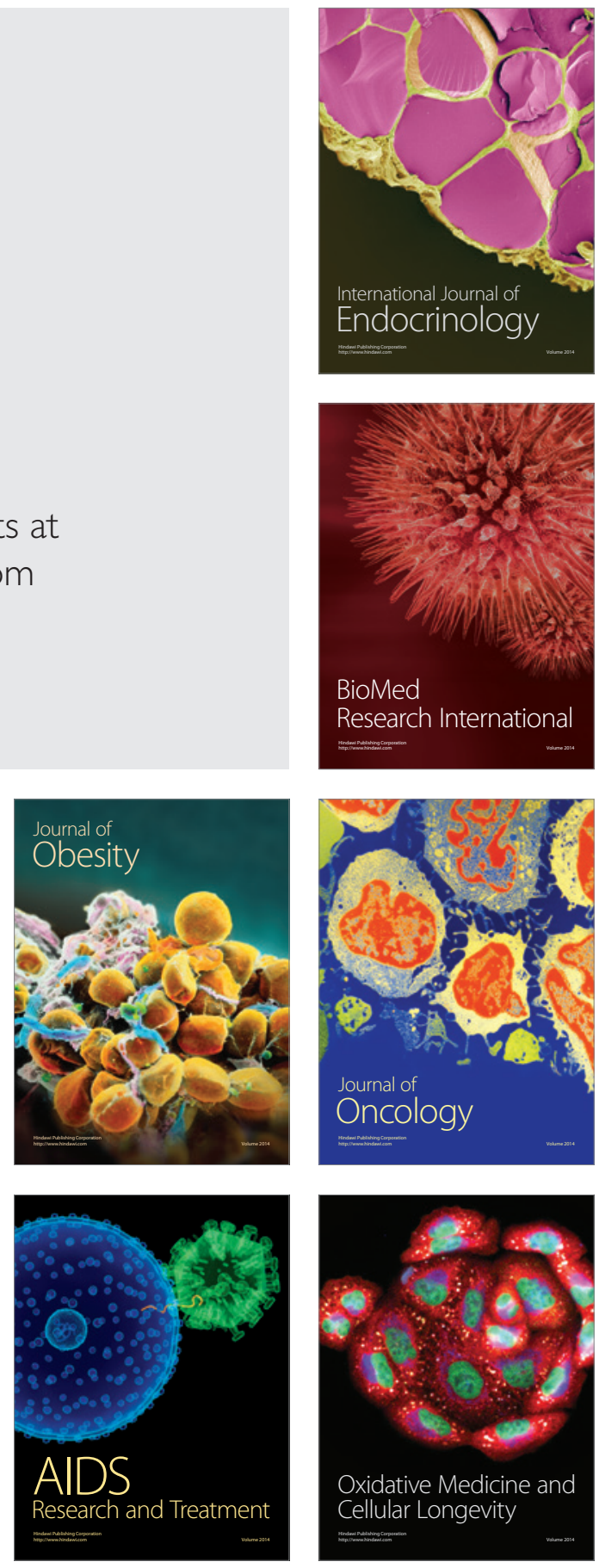